\title{
NEW UPPER BOUNDS ON THE PROBABILITY OF EVENTS BASED ON GRAPH STRUCTURES
}

\author{
BÉLA VIZVÁRI
}

Abstract. We present upper bounds for the probability of the union of events based on the individual probabilities and joint probabilities of pairs. The bounds generalize Hunter's upper bound and can be interpreted as objective function values corresponding to feasible solutions of the dual of the Boolean probability bounding LP.

Mathematics subject classification (2000): 90C35, 90C90, 90C05.

Key words and phrases: probability bound, graph, dual feasible solution.

\section{REFERENCES}

[1] C. E. BonfERroni, Teoria statistica delle classi e calcolo delle probabilitá, Volume in onore di Riccardo Dalla Volta, Universitá di Firenze, (1937), 1-62.

[2] G. Boole, Laws of Thought, American reprint of 1854 edition, Dover, New York, (1854).

[3] G. Boole, Of Propositions Numerically Definite, in Transactions of Cambridge Philosophical Society, Part II, XI, (1868), reprinted as Study IV in the next reference.

[4] G. Boole, Collected Logical Works, Vol. I. Studies in Logic and Probability, R. Rhees (ed.), Open Court Publ. Co., LaSalle, Ill., (1952).

[5] E. Boros, A. PrÉKopa, Closed Form Two-Sided Bounds for Probabilities that Exactly $r$ and at Least $r$ out of $n$ Events Occur, Mathematics of Operations Research, 14, (1989), 317-342.

[6] D. A. Dawson, SAnKoff, An Inequality for Probabilities, Proceedings of the American Mathematical Society, 18, (1967), 504-507.

[7] A. FRANK, Edge-Connection of Graphs, and Hypergraphs, FINF, E. Gyфri (ed.), János Bolyai Mathematical Society, to appear (2004).

[8] J. Galambos,Bonferroni Inequalities, Annals of Probability, 5, (1977), 577-581.

[9] Th. Hailperin, Best Possible Inequalities for the Probability of a Logical Function of Events, The American Mathematical Monthly, 72, (1965), 343-359.

[10] D. HunTER, An upper bound for the probability of a logical function of events, J. Appl. Prob., 13, (1976), 597-603.

[11] H. KuAi, F. Alajaji AND G. TAKahara, A Lower Bound on the Probability of a Finite Union of Events, Discrete Applied Mathematics, to appear, (1999).

[12] S. Kounias, J. Marin, Best Linear Bonferroni Bounds, SIAM J. on Applied Mathematics, 30, (1976), 307-323.

[13] S. M. KWEREL, Most Stringent Bounds on Aggregated Probabilities of Partially Specified Dependent Probability Systems, J. Am. Statist. Assoc., 70, (a) (1975), 472-479.

[14] S. M. KWEREL, Bounds on Probability of a Union and Intersection of $m$ Events, Advances of Applied Probability, 7, (b) (1975), 431-448.

[15] A. PRÉKOPA, Boole-Bonferroni Inequalities and Linear Programming, Operations Research, 36, (1988), 145-162.

[16] A. PRÉKOPA, Sharp Bounds on Probabilities Using Linear Programming, Operations Research, 38, (1990), 227-239. 
[17] A. PRÉKOPA, Inequalities on Expectations Based on the Knowledge of Multivariate Moments, in Stochastic Inequalities, M. Shaked, and Y.L. Tong (eds.), Institute of Mathematical Statistics, Lecture Notes - Monograph Series, 22,(1992), 309-331.

[18] A. PRÉKOPA, Bounds on Probabilities and Expectations Using Multivariate Moments of Dicrete Distributions, Studia Sci. Math. Hung., 34, (1998), 349-378.

[19] A. PRÉKOPA, L. GAO, Bounding Probability of Events by Aggregation and Disaggregation in Linear in Linear Programs, Discrete Applied Mathematics, 145, (2005), 444-454.

[20] A. PrÉKOPA, B. VizvÁRI AND G. REgфS, Lower and Upper Bounds on Probabilities of Boolean Functions of Events, RUTgers Center of Operations Research (RUTCOR), RRR 36-95.

[21] Y. S. SATHE, M. PRADHAN AND S. P. SHAH, Inequalities for the Probability of the Occurrence of at Least $m$ out of $n$ Events, Journal of Applied Probability, 17, (1980), 1127-1132.

[22] P. VenEZIANI, Upper Bounds on the Probability of the Union of Events, RUTgers Center of Operations Research (RUTCOR), RRR 14-2000. 